University of Wollongong

Research Online

National Institute for Applied Statistics

Faculty of Engineering and Information

Research Australia Working Paper Series

Sciences

2015

\title{
Hot enough for you? A spatial exploratory and inferential analysis of North American climate-change projections
}

Noel Cressie

University of Wollongong

Emily L. Kang

University of Cincinnati

Follow this and additional works at: https://ro.uow.edu.au/niasrawp

\section{Recommended Citation}

Cressie, Noel and Kang, Emily L., Hot enough for you? A spatial exploratory and inferential analysis of North American climate-change projections, National Institute for Applied Statistics Research Australia, University of Wollongong, Working Paper 01-15, 2015, 17.

https://ro.uow.edu.au/niasrawp/21

Research Online is the open access institutional repository for the University of Wollongong. For further information contact the UOW Library: research-pubs@uow.edu.au 


\title{
Hot enough for you? A spatial exploratory and inferential analysis of North American climate-change projections
}

\begin{abstract}
Climate models have become the primary tools for scientists to project climate change into the future and to understand its potential impact. Continental-scale General Circulation Models (GCMs) oversimplify the regional climate processes and geophysical features such as topography and land cover. Since the consequences of local/regional climate change are particularly relevant to natural-resource management and environmental-policy decisions, Regional Climate Models (RCMs) have been developed to produce high-resolution outputs on scales of $50 \mathrm{~km}$ and smaller. RCMs can simulate three-hourly "weather" over long time periods and generate a vast array of outputs, from which long-run averages are commonly used as a summary of how a climate model approximates a region's climate. With anthropogenic forcings incorporated, RCMS provide a means to assess a combination of natural and anthropogenic influences on climate variability.

The North American Regional Climate Change Assessment Program (NARCCAP) ran regional Climate Models (RCMs) 60 years into the future for 11,760 regions in North America, each of which is approximately $50 \mathrm{~km} \times 50 \mathrm{~km}$ in area. Using the 94,080 temperature changes projected to 2070 for all regions, for two RCMs, and for the four seasons, both an exploratory and an inferential spatial analysis is presented; the inferential analysis is based on a Bayesian hierarchical model. Videos show regions of North America that attain or exceed temperature-change thresholds as a function of increasing threshold. The preponderance of our results throughout all regions of North America is one of warming by 2070, usually more (and sometimes much more) than $2{ }^{\circ} \mathrm{C}$.
\end{abstract}




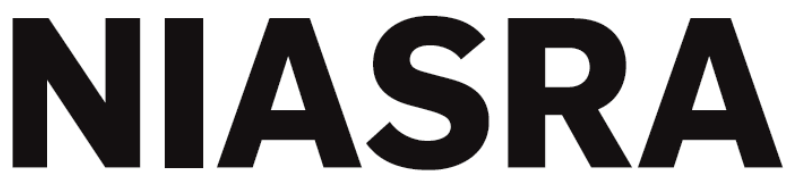

NATIONAL INSTITUTE FOR APPLIED STATISTICS RESEARCH AUSTRALIA

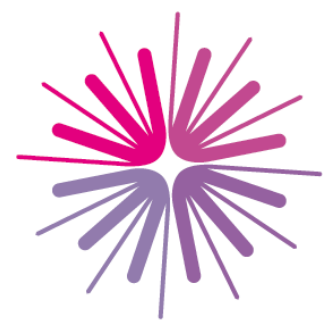

\title{
National Institute for Applied Statistics Research Australia
}

\author{
University of Wollongong
}

\section{Working Paper}

\section{1-15}

Hot Enough for You? A Spatial Exploratory and Inferential Analysis of North American Climate-Change Projections

\author{
Noel Cressie and Emily L. Kang
}




\title{
Hot Enough for You? A Spatial Exploratory and Inferential Analysis of North American Climate-Change Projections
}

\author{
Noel Cressie ${ }^{1} \quad$ Emily L. Kang ${ }^{2}$
}

December 30, 2014

\begin{abstract}
Climate models have become the primary tools for scientists to project climate change into the future and to understand its potential impact. Continental-scale General Circulation Models (GCMs) oversimplify the regional climate processes and geophysical features such as topography and land cover. Since the consequences of lo$\mathrm{cal} /$ regional climate change are particularly relevant to natural-resource management and environmental-policy decisions, Regional Climate Models (RCMs) have been developed to produce high-resolution outputs on scales of $50 \mathrm{~km}$ and smaller. RCMs can simulate three-hourly "weather" over long time periods and generate a vast array of outputs, from which long-run averages are commonly used as a summary of how a climate model approximates a region's climate. With anthropogenic forcings incorporated, RCMS provide a means to assess a combination of natural and anthropogenic influences on climate variability.

The North American Regional Climate Change Assessment Program (NARCCAP) ran regional Climate Models (RCMs) 60 years into the future for 11,760 regions in North America, each of which is approximately $50 \mathrm{~km} \times 50 \mathrm{~km}$ in area. Using the 94,080 temperature changes projected to 2070 for all regions, for two RCMs, and for the four seasons, both an exploratory and an inferential spatial analysis is presented; the inferential analysis is based on a Bayesian hierarchical model. Videos show regions of North America that attain or exceed temperature-change thresholds as a function of increasing threshold. The preponderance of our results throughout all regions of North America is one of warming by 2070, usually more (and sometimes much more) than $2^{\circ} \mathrm{C}$.
\end{abstract}

Keywords: ESDA; PROT function; spatial hierarchical model; SPOT function; temperaturechange projections

\section{Introduction}

Climate models have become primary tools for scientists to project future climate change and to understand its potential impact. Since the late 1960s, Atmosphere-Ocean General Circulation Models (GCMs) have been developed to simulate the climate over the entire globe. GCMs couple an atmospheric model with an oceanic model to simulate components of the global climate system, such as circulations and forcings. Due to model complexity and limitations of computational resources, GCMs are restricted to generate outputs on coarse spatial scales, typically 200 to $500 \mathrm{~km}$. Additionally, due to their global perspective, GCMs oversimplify the regional climate processes and geophysical features such

\footnotetext{
${ }^{1}$ NIASRA, University of Wollongong NSW 2522, Australia

${ }^{2}$ Department of Mathematical Sciences, University of Cincinnati, Cincinnati OH 45221-0025
} 
as topography and land cover. Since local/regional climate effects are more relevant to natural-resource management and environmental-policy decisions, Regional Climate Models (RCMs) have been developed to produce high-resolution outputs on scales of $50 \mathrm{~km}$ and smaller. Nevertheless, RCMs need initial conditions and time-dependent boundary conditions, which are typically provided by a GCM; this is sometimes referred to as "dynamic downscaling" of the GCM outputs (e.g., Fennessy and Shukla 2000; Xue et al. 2007).

Essentially, GCMs and RCMs are a series of discretized differential equations that attempt to represent physical relationships such as the flows of energy and water within and between the atmosphere, oceans, land, sea ice, and so forth. Using differential equations that describe the physical dynamics, RCMs can simulate three-hourly "weather" over long time periods and generate a vast array of outputs, from which the long-run average is commonly used as a summary of how a climate model approximates a region's climate. With anthropogenic forcings incorporated, climate models can be run under different scenarios (e.g., various $\mathrm{CO}_{2}$ levels). Consequently, natural and anthropogenic influences on climate variability can be assessed.

GCMs and RCMs are complicated to build, and they are deterministic (i.e., two runs of the same model under identical conditions produce identical outputs). Nevertheless, there are various sources of uncertainty that accompany such models. For example, there may be uncertainty in assumptions about interaction between atmospheric circulation and orography, about discretization, or about parameterizations of the physical-forcing processes. To obtain a better understanding of such uncertainties, climate scientists carry out experiments with multiple runs of multiple models. In this article, we consider climate-model output from the North American Regional Climate Change Assessment Program (NARCCAP; Mearns et al. 2009), and we concentrate on RCMs with the same initial and boundary conditions (supplied by the same GCM) and the same greenhouse-gas (GHG) forcing (supplied by a scenario that matches most closely current GHG emissions; Nakicenovic et al. 2000).

NARCCAP is an international program whose goal is to produce high-resolution climate simulations for current and future time periods. Thus, there is an opportunity to investigate spatial variability of regional-scale projections of future climate and to generate temperature-change scenarios for use in impacts research. NARCCAP produces this high-resolution (approximately $50 \mathrm{~km}$ ) climate-output data for six RCMs built for the North American region that includes the US, Canada, northern Mexico, and the oceans nearby (Mearns et al. 2009).

NARCCAP Phase I explores the variability in RCM outputs for the current period, where the six RCMs were run with common boundary conditions provided by the NCEPDOE Reanalysis II data (e.g., Kanamitsu et al. 2002). NARCCAP Phase II involves not only multiple RCMs but also runs with different boundary conditions provided by different GCMs.

In Phase II, these six RCMs are coupled with four different GCMs and were run not only for the current period (1971-2000) but also for a future period (2041-2070). Thus, temperature-change projections are available from the Phase II experiment. In both Phase I and Phase II, the same greenhouse-gas emissions scenario (SRES A2; Nakicenovic et al. 2000) were used.

A number of different aspects of the NARCCAP data have already been analyzed (e.g., Kaufman and Sain 2010; Salazar et al. 2011; Kang et al. 2012; Kang and Cressie 2013). In this article, attention is paid to the seasonal climate projections, particularly the Boreal winter, reasoning that projected warming over North America might be most clearly seen in the months of December, January, and February; see Kang and Cressie (2013). 
In this article, we consider a subset of the output from the Phase II runs, namely the average surface yearly temperatures and the average surface Boreal-winter (December, January, and February) temperatures, for the current period (1971-2000) and for the future period (2041-2070). We analyze the output produced by the two RCMs, namely CRCM and RCM3, with the same GCM, namely CGCM3, providing the boundary conditions; for further details on these and other climate models used in NARCCAP, see Kang and Cressie (2013). The RCM outputs for the current and future periods were given on an approximately $50 \mathrm{~km} \times 50 \mathrm{~km}$ NARCCAP grid of $98 \times 120$ pixels, resulting in 11,760 NARCCAP pixels. These are "big data"; there are a total of 5.6 million surface temperature values used in the exploratory and inferential analyses presented in this article.

Section 2 gives exploratory spatial data analyses (ESDA) of the subset of Phase II output under consideration. The SPOT function is introduced and illustrated on the yearly and winter temperature-change data. Section 3 presents a Bayesian hierarchical spatial model of the NARCCAP data used in Sect. 2; and inferential analysis is given in Sect. 4, where the PROT function is introduced. A discussion and various conclusions are given in Sect. 5.

\section{Exploratory Spatial Data Analysis and the SPOT Func- tion}

Consider the current and future 30-year-averaged seasonal surface temperature fields from the $i$-th RCM for the $j$-th Boreal season, where $i=1,2$, and $j=1$ (spring: March, April, May), $i=2$ (summer: June, July, August), $j=3$ (autumn: September, October, November), $j=4$ (winter: December, January, February). Define

$$
\begin{aligned}
Z_{i j}^{\text {current }}\left(\mathbf{s}_{l}\right) & \equiv(1 / 30) \sum_{t=1971}^{2000} Z_{i j}\left(\mathbf{s}_{l} ; t\right) \\
Z_{i j}^{\text {future }}\left(\mathbf{s}_{l}\right) & \equiv(1 / 30) \sum_{t=2041}^{2070} Z_{i j}\left(\mathbf{s}_{l} ; t\right),
\end{aligned}
$$

where $l=1, \ldots, 11760$ represents the index of the $l$-th pixel on the $98 \times 120$ NARCCAP grid that is superimposed on North America. Here, $Z_{i j}\left(\mathbf{s}_{l} ; t\right)$ is the corresponding region's average surface temperature for the $i$-th RCM and the $j$-th season in year $t$. In all, there are 5.6 million spatio-temporal $Z$-values used in the two definitions given above.

In this article, we are particularly interested in temperature change for the winter $(j=4)$ and for the whole year (averaged over $j=1,2,3,4)$. Further, we mostly consider the output of the RCMs averaged over the models $i=1,2$, although some discussion of the between-model variability is given in Sect. 5 ; it will be seen to be small (about $5 \%$ of the total variability). Define the temperature-change projections,

$$
\begin{aligned}
& D^{w i}\left(\mathbf{s}_{l}\right) \equiv(1 / 2) \sum_{i=1}^{2} Z_{i 4}^{\text {future }}\left(\mathbf{s}_{l}\right)-(1 / 2) \sum_{i=1}^{2} Z_{i 4}^{\text {current }}\left(\mathbf{s}_{l}\right) \\
& D^{y r}\left(\mathbf{s}_{l}\right) \equiv(1 / 8) \sum_{i=1}^{2} \sum_{j=1}^{4} Z_{i j}^{\text {future }}\left(\mathbf{s}_{l}\right)-(1 / 8) \sum_{i=1}^{2} \sum_{j=1}^{4} Z_{i j}^{\text {current }}\left(\mathbf{s}_{l}\right) .
\end{aligned}
$$

These represent projections of (30-year-averaged) North American surface temperature changes, projected out to 2070. Each of (1) and (2) involves 11,760 data defined on the 
NARCCAP grid. This spatial context means that visualizations through mapping can (and should) be part of any exploratory data analysis of temperature-change projections obtained from NARCCAP.

It can be seen from Fig. 1 that the temperature changes are consistently positive for both winter output and yearly output. That is, it is projected to be considerably warmer by 2070 over the entire North American region. Generally speaking, the warming effect is stronger over land compared to that over ocean. It is also apparent that the warming effect during the winter in the northern part of the North America region is particularly strong, especially in the Hudson Bay area.

These visualizations represent "map views," which can be enhanced by a "multivariate view," namely an $x-y$ plot of winter output versus yearly output. Figure 1 also shows a plot of the 11760 points $\left\{\left(D^{y r}\left(\mathbf{s}_{l}\right), D^{w i}\left(\mathbf{s}_{l}\right)\right): l=1, \ldots, 11760\right\}$. As expected, the winter temperature changes are more extreme than the yearly ones, since the yearly changes are averages over all seasons. Obviously, people and communities live through seasons, and hence it is highly relevant to analyze seasonal behavior as well as yearly behavior.

\section{$\overline{\text { Figure } 1 \text { here }}$}

To quantify these impressions, a function is introduced that plots the spatial proportion (of pixels) over a threshold as a function of that threshold. This SPOT (Spatial Proportion Over Threshold) function is defined as follows.

$$
\begin{array}{ll}
T^{w i}(k) \equiv(1 / n) \sum_{l=1}^{n} I\left(D^{w i}\left(\mathbf{s}_{l}\right)>k\right) ; & -\infty<k<\infty \\
T^{y r}(k) \equiv(1 / n) \sum_{l=1}^{n} I\left(D^{y r}\left(\mathbf{s}_{l}\right)>k\right) ; & -\infty<k<\infty,
\end{array}
$$

where $n=11760$ is the number of spatial regions being considered. A very powerful technique in exploratory spatial data analysis is to "link and brush," namely to "paint" on a map of North America all those regions $\left\{\mathbf{s}_{l}\right\}$ whose projected temperature change exceeds the threshold $k$. Figure 2 shows a video of the "SPOT view" and "map view," linked, as $k$ increases from $1.0^{\circ} \mathrm{C}$ to $7.6^{\circ} \mathrm{C}$ in units of $0.2^{\circ} \mathrm{C}$. Both $T^{w i}(\cdot)$ and $T^{y r}(\cdot)$ are shown. The linking of the SPOT view and the map view shows where and what proportion of pixels exceed a range of thresholds.

\section{$\overline{\text { Figure } 2 \text { here }}$}

The role of exploratory spatial data analysis is to generate ideas about specific sources of variability, which can be followed up by fitting statistical models and carrying out statistical inference. Here, spatial variation is an obvious source of variability, which we have already seen in Figs. 1 and 2. In the next section, we quantify the variation of the RCMs' outputs with a stochastic model and use it to address the questions, "Is climate change real?" and "What are the projected temperature changes along with measures of their certainty?" Critically, this is done with a paradigm that expresses uncertainties through probability distributions.

\section{Spatial Hierarchical Model of Temperature Change by 2070}

Recall the definitions (1) and (2) obtained from the output of a collection of (here two) RCMs. It is not expected that in 2070, temperatures will increase exactly by any of these 
amounts. While the output of an RCM is deterministic, there is uncertainty about how continuous-time, continuous-space partial differential equations should be discretized onto grids, along with where and how forcing terms (e.g., greenhouse gases) are introduced. Thus, an RCM's output could be thought of as a single realization of many possible, which are governed by a probability distribution (e.g., Kaufman and Sain 2010; Sain et al. 2011; Salazar et al. 2011; Kang et al. 2012; Kang and Cressie 2013). It should be noted that geostatistics takes exactly the same approach when making inferences on an ore body, even though the mineralization of a given ore body was a unique event.

\subsection{Introduction to Hierarchical Statistical Modeling}

A hierarchical statistical model is one where the model can be broken down into at least two levels: The data model and the process model. A Bayesian hierarchical statistical model involves three levels: The data model, the process model and, additionally, the parameter (or prior) model. When these models are multiplied together, they form the joint distribution of the data and the process/parameters (e.g., Berliner 1996). The data model describes the conditional probability distribution of the data, given parameters and an unobserved (hidden) process. The process model describes the conditional probability distribution of the hidden process given its parameters. The parameter model puts a "prior" distribution on the parameters themselves.

In the application presented here, the data model describes the long-run average differences between future and current climate-model runs, where the hidden climate process is made up of the projected temperature changes by season and RCM. The process model incorporates the Spatial Random Effects (SRE) model (Cressie and Johannesson 2008), which is an effective way to reduce the dimensionality of the problem from approximately 100,000 to less than 100. Prior distributions are assigned to parameters, which constitutes the parameter model. The ultimate goal is to obtain the posterior distribution, namely the joint distribution of the unknowns in the hierarchical statistical model (i.e., process and parameters), given the data. The predictive distribution is simply the marginal distribution of the unknown process given the data. Using Bayes' Theorem, the posterior distribution is proportional to the product of the data, process, and parameter models. Simulation procedures, such as Markov chain Monte Carlo (MCMC) methods, are used here to obtain the predictive distribution of any part of the process (given the data). Further details on the data/process and data/process/parameters hierarchical framework can be found in Cressie and Wikle (2011, Chap. 2).

There are several advantages to using a hierarchical statistical approach. First, nonhierarchical models with just a few parameters generally do not fit the data well. Moreover, non-hierarchical models with many parameters may fit the data well but tend to "over-fit" and may not be useful for predictive purposes. In contrast, hierarchical statistical models can often fit the data well with just a few parameters, and they also do well for predicting the hidden process (at both observed and unobserved parts of the process).

\subsection{Data Model}

This part of the hierarchical statistical model usually incorporates the component of variability due to measurement error. But, it can also capture other sources of variability extraneous to the hidden process of interest, such as spatio-temporal interactions. Recall the definition of $Z_{i j}\left(\mathbf{s}_{l} ; t\right)$ as the $l$-th region's average surface temperature for the $i$-th RCM and the $j$-th season in year $t$. 
For $t=2041, \ldots, 2070$, write

$$
Z_{i j}\left(\mathbf{s}_{l} ; t\right)-Z_{i j}\left(\mathbf{s}_{l} ; t-70\right)=Y_{i j}\left(\mathbf{s}_{l}\right)+e_{i j}(\mathbf{s} ; t),
$$

where $Y_{i j}(\cdot)$ is the purely spatial process of temperature change by 2070 , and $e_{i j}(\cdot ; t)$ is an independent smaller-scale component of variation capturing spatio-temporal interaction. It is assumed in (5) that $Y_{i j}$ carries all the spatial dependence in the temperaturechange field and that $e_{i j}$ has none. Further, $e_{i j}$ has mean zero and, independently for $t=2041, \ldots, 2071$,

$$
e_{i j}(\cdot ; t) \sim \operatorname{Gau}\left(0, V_{i j}(\cdot) \sigma_{e}^{2}\right) .
$$

Define the 30-year average temperature difference,

$$
D_{i j}\left(\mathbf{s}_{l}\right) \equiv(1 / 30) \sum_{t=2041}^{2070}\left\{Z_{i j}\left(\mathbf{s}_{l} ; t\right)-Z_{i j}\left(\mathbf{s}_{l} ; t-70\right)\right\},
$$

and hence, from $(5), D_{i j}(\cdot)$ can be written as

$$
D_{i j}(\cdot)=Y_{i j}(\cdot)+\varepsilon_{i j}(\cdot),
$$

where the error term $\varepsilon_{i j}$ has no spatial dependence, and

$$
\varepsilon_{i j}\left(\mathbf{s}_{l}\right) \sim \operatorname{Gau}\left(0, V_{i j}\left(\mathbf{s}_{l}\right) \sigma_{\varepsilon}^{2}\right),
$$

for $\sigma_{\varepsilon}^{2}=(1 / 30) \sigma_{e}^{2}$. The spatial heterogeneity of the error term is captured by $V_{i j}(\cdot)$, which is given by

$$
V_{i j}\left(\mathbf{s}_{l}\right) \equiv(1 / 29) \sum_{t=2041}^{2070}\left\{Z_{i j}\left(\mathbf{s}_{l} ; t\right)-Z_{i j}\left(\mathbf{s}_{l} ; t-70\right)-D_{i j}\left(\mathbf{s}_{l}\right)\right\}^{2} .
$$

Equation (7) is the data model. The goal is to filter out the error term $\varepsilon_{i j}$ and to make inference on the hidden spatial process $Y_{i j}$; this is the formulation developed by Kang and Cressie (2013).

In this article, we are interested in temperature changes for the winter and for the whole year. From (1), (2), and (7),

$$
\begin{aligned}
& D^{w i}\left(\mathbf{s}_{l}\right)=Y^{w i}\left(\mathbf{s}_{l}\right)+(1 / 2)\left(\sum_{i=1}^{2} \varepsilon_{i 4}\left(\mathbf{s}_{l}\right)\right) \\
& D^{y r}\left(\mathbf{s}_{l}\right)=Y^{y r}\left(\mathbf{s}_{l}\right)+(1 / 8)\left(\sum_{i=1}^{2} \sum_{j=1}^{4} \varepsilon_{i j}\left(\mathbf{s}_{l}\right)\right),
\end{aligned}
$$

for $l=1, \ldots, 11760 ;$ and in $(8)$ and $(9)$,

$$
Y^{w i}(\cdot) \equiv(1 / 2) \sum_{i=1}^{2} Y_{i 4}(\cdot) ; \quad Y^{y r}(\cdot) \equiv(1 / 8) \sum_{i=1}^{2} \sum_{j=1}^{4} Y_{i j}(\cdot) .
$$

Inference will be on the two hidden spatial processes, $Y^{w i}$ and $Y^{y r}$, which from (11) are defined in terms of $Y_{i j}$ given in (7). 


\subsection{Process and Parameter Models}

The variability in the temperature-change process $Y_{i j}$ is due to climate-model differences, seasonal differences, and spatial variability, This can be described through the decomposition

$$
Y_{i j}(\cdot)=\mu(\cdot)+a_{i}(\cdot)+b_{j}(\cdot)+(a b)_{i j}(\cdot),
$$

where $\mu(\cdot)$ represents the baseline temperature change; $a_{i}(\cdot)$ is the extra component due to the $i$-th RCM; $b_{j}(\cdot)$ is the extra component due to the $j$-th season; and the terms $\left\{(a b)_{i j}(\cdot)\right\}$ capture the RCM-season interaction and typically exhibit smaller-scale variability.

All the components on the right-hand side of (12) are spatial processes, each with $n=11760$ elements. The spatial covariance matrices of these processes are $11760 \times 11760$ and generally too large to be inverted, so Kang and Cressie (2013) proposed the following dimension reduction,

$$
Y_{i j}(\cdot)=\mu(\cdot)+\mathbf{S}(\cdot)^{\prime} \boldsymbol{\eta}_{i j},
$$

where $\mu(\cdot)$ is a deterministic trend that may depend on covariates through $\mu(\cdot)=\mathbf{x}(\cdot)^{\prime} \boldsymbol{\beta}$; $\mathbf{S}(\cdot)$ is a known $r$-dimensional vector defined by $r$ spatial basis functions such that $r \ll n$; and the $r$-dimensional, zero-mean random vector of coefficients, $\boldsymbol{\eta}_{i j}$, is decomposed into independent zero-mean components according to

$$
\boldsymbol{\eta}_{i j}=\boldsymbol{\delta}_{i}+\gamma_{j}+\boldsymbol{\zeta}_{i j}
$$

For $i=1,2$, Kang and Cressie (2013) fitted the model defined by the random effects due to

$\mathrm{RCM}: \quad \boldsymbol{\delta}_{i} \sim \operatorname{Gau}\left(\mathbf{0}, \mathbf{K}_{1}\right)$

Season: $\quad \boldsymbol{\gamma}_{j} \sim \operatorname{Gau}\left(\mathbf{0}, \mathbf{K}_{2}\right), j=1,3 ; \quad \boldsymbol{\gamma}_{j} \sim \operatorname{Gau}\left(\mathbf{0}, \mathbf{K}_{3}\right), j=2,4$

Interaction: $\quad \boldsymbol{\zeta}_{i j} \sim \operatorname{Gau}\left(\mathbf{0}, \mathbf{K}_{4}\right), j=1,3 ; \quad \boldsymbol{\zeta}_{i j} \sim \operatorname{Gau}\left(\mathbf{0}, \mathbf{K}_{5}\right), j=2,4$,

which allows the covariance matrices to differ between the mild spring and autumn seasons and the more extreme summer and winter seasons. The spatial basis functions chosen by Kang and Cressie (2013) captured different scales of variability, elevations and presence/absence of pixels on land, the Great Lakes, Hudson Bay, and coastlines. This resulted in $r=85$ spatial basis functions.

Equations (13) and (14) represent the process model, and (13) is called a Spatial Random Effects (SRE) model; see Cressie and Johannesson (2008). Finally, the parameter models are set out in full detail in Kang and Cressie (2013, Sect. 3.3), to which we refer the interested reader.

\subsection{Predictive Distributions of 11,760 Temperature-Change Values}

Very simply, the data model is the distribution of $D$ given $Y$ (first level), the process model is the distribution of $Y$ (second level), and the predictive distribution is the distribution of

$$
[Y \text { given } D] \propto[D \text { given } Y] \times[Y]
$$

by Bayes' Theorem. The extra level in a Bayesian hierarchical model is the parameter model, for which the generalization of Bayes' Theorem is straightforward. This third level does not change the need for obtaining a predictive distribution of $Y$ given $D$.

Fundamentally, inference on the process is conditional on the data. In the NARCCAP context, $Y$ is made up of 94,080 process values $\left\{Y_{i j}(\cdot)\right\}, D$ is made up of 94,080 data values 
$\left\{D_{i j}(\cdot)\right\}$, and Bayes' Theorem is used to obtain the predictive distribution of $\left\{Y_{i j}(\cdot)\right\}$ given $\left\{D_{i j}(\cdot)\right\}$. However, with the large number of data values and process values, it is generally computationally infeasible to obtain the predictive distribution, and so some form of dimension reduction is needed.

Randomness appears in the data model (7) very simply through independent error, and it appears in the process model (13) and (14) through the $r$-dimensional $(r=85)$ vectors $\left\{\boldsymbol{\eta}_{i j}: i=1,2 ; j=1,2,3,4\right\}$. Critically, if the $85 \times 2 \times 4=680$ process values in (14) can be inferred, then all of $\left\{Y_{i j}(\cdot): i=1,2 ; j=1,2,3,4\right\}$ can be inferred. Consequently, dimension-reduced inference is obtained through the predictive distribution of

$$
\left\{\boldsymbol{\eta}_{i j}\right\} \text { given }\left\{D_{i j}\left(\mathbf{s}_{l}\right): i=1,2 ; j=1,2,3,4 ; l=1, \ldots, 11760\right\} .
$$

Kang and Cressie (2013) give the details of an MCMC algorithm that is used to sample from the predictive distribution of $\left\{Y_{i j}(\cdot)\right\}$ given $\left\{D_{i j}(\cdot)\right\}$ based on the dimension reduction afforded by the SRE model in (13). Thus, samples from the predictive distribution of

$$
Y^{w i}(\cdot) \text { given }\left\{D_{i j}(\cdot)\right\} ; \quad Y^{y r}(\cdot) \text { given }\left\{D_{i j}(\cdot)\right\},
$$

are straightforwardly generated. All inferences presented in this article are obtained from such predictive distributions. In the next section, inference on an individual region's temperature change as well as inferential analogues to the exploratory SPOT functions (Sect. 2) are presented.

\section{Spatial Inference and the PROT Function}

In Sect. 3.4, it was explained how MCMC samples from the predictive distribution of $\left\{Y_{i j}(\cdot)\right\}$ given the data $\left\{D_{i j}(\cdot)\right\}$ can be used to obtain samples from the predictive distribution of $Y^{w i}(\cdot)$ and $Y^{y r}(\cdot)$ given the data $\left\{D_{i j}(\cdot)\right\}$. For example, consider the pixel $\mathbf{s}_{0}$ containing the National Center for Atmospheric Research (NCAR) Mesa Lab in Boulder, $\mathrm{CO}$, where the NARCCAP project was conducted. The "NCAR" pixel's predictive distributions, of $Y^{w i}\left(\mathbf{s}_{0}\right)$ given the data and of $Y^{y r}\left(\mathbf{s}_{0}\right)$ given the data, are shown in Fig. 3. Notice that the predictive distribution of temperature change is centered at about $2^{\circ} \mathrm{C}$ for the winter season and at about $2.7^{\circ} \mathrm{C}$ for the whole year. In both situations, there is a predictive probability of 1.000 that climate change will be greater than $1.5^{\circ} \mathrm{C}$ by 2070 .

\section{Figure 3 here}

The predictive distribution of any function of $\left\{Y_{i j}(\cdot)\right\}$, not just a linear function, can be obtained immediately from the MCMC samples. For the $l$-th pixel located at $\mathbf{s}_{l}$, define the functions

$$
I\left(Y^{w i}\left(\mathbf{s}_{l}\right)>k\right) ; \quad I\left(Y^{y r}\left(\mathbf{s}_{l}\right)>k\right),
$$

for $-\infty<k<\infty$. Notice that the indicator function $I(\cdot)$ in $(15)$ is nonlinear and has a range of $\{0,1\}$. This function is of interest because beyond certain temperature thresholds, insects may thrive, crops may fail, and native plant species may relocate.

One convenient summary of the predictive distribution is its mean. In terms of the data $D$ and the process $Y$ introduced in Sect. 3.4, suppose one wishes to make inference on the (possibly nonlinear) function $g(Y)$. Then an often-used predictor is the predictive mean

$$
\hat{g} \equiv \mathrm{E}(g(Y) \mid D)
$$


which is obviously a function of the data. In the case of $g(\cdot)$ defined by (15), the predictors for the winter season and for the whole year are, in obvious notation,

$$
\begin{aligned}
& \hat{g}^{w i}\left(\mathbf{s}_{l} ; k\right) \equiv \operatorname{Pr}\left(Y^{w i}\left(\mathbf{s}_{l}\right)>k \mid\left\{D_{i j}(\cdot)\right\}\right) \\
& \hat{g}^{y r}\left(\mathbf{s}_{l} ; k\right) \equiv \operatorname{Pr}\left(Y^{y r}\left(\mathbf{s}_{l}\right)>k \mid\left\{D_{i j}(\cdot)\right\}\right),
\end{aligned}
$$

for $l=1, \ldots, 11760$. Definitions (16) and (17) are the predictive probabilities over a threshold as a function of the threshold. This PROT (PRedictive probability Over Threshold) function is formally defined as follows: For $l=1, \ldots, 11760$,

$$
\begin{aligned}
& P^{w i}\left(k ; \mathbf{s}_{l}\right) \equiv \hat{g}^{w i}\left(\mathbf{s}_{l} ; k\right) ; \quad-\infty<k<\infty \\
& P^{y r}\left(k ; \mathbf{s}_{l}\right) \equiv \hat{g}^{y r}\left(\mathbf{s}_{l} ; k\right) ; \quad-\infty<k<\infty \text {. }
\end{aligned}
$$

Notice that the PROT functions depend on the location $\mathbf{s}_{l}$ in the spatial domain, unlike the SPOT functions. Hence, for each threshold $k$, a choropleth map can be made of the predictive probabilities that projected temperature changes will exceed $k$. Figure 4 shows a video of maps based on the PROT functions, $\left\{P^{w i}\left(k ; \mathbf{s}_{l}\right): l=1, \ldots, 11760\right\}$ and $\left\{P^{y r}\left(k ; \mathbf{s}_{l}\right): l=1, \ldots, 11760\right\}$, as $k$ increases from $1.0^{\circ} \mathrm{C}$ to $6.8^{\circ} \mathrm{C}$ in increments of $0.2^{\circ} \mathrm{C}$.

\section{Figure 4 here}

The PROT values in (18) and (19) are generated for each NARCCAP pixel, but they could also be generated for a coarser resolution of the NARCCAP region. Now inference would be on average temperature change over, say, a coarser-resolution block B. For example, for winter, the (nonlinear) function of interest is

$$
I\left(\left(\sum_{\mathbf{s} \in B} Y^{w i}(\mathbf{s}) / \sum_{\mathbf{s} \in B} 1\right)>k\right) .
$$

Then inference proceeds exactly as before, based on MCMC samples from the predictive distribution of $\left\{\boldsymbol{\eta}_{i j}\right\}$ given the data.

\section{Discussion and Conclusions}

Using the spatial exploratory data analysis described in Sect. 2 and the inference described in Sect. 4, the between-model variability of the RCMs can be investigated. Figure 5 shows two maps of the differences of the two model outputs, one for winter and one for the whole year. That is,

$$
D_{14}(\cdot)-D_{24}(\cdot) ; \quad(1 / 4) \sum_{j=1}^{4} D_{1 j}(\cdot)-(1 / 4) \sum_{j=1}^{4} D_{2 j}(\cdot)
$$

are maps for winter and the whole year, respectively. The scale on the maps, in degrees $\mathrm{C}$, indicates very little difference between the two RCMs.

\section{$\overline{\text { Figure } 5 \text { here }}$}

To formalize this impression, consider the following measure of between-model variability, defined in terms of the hidden process $\left\{Y_{i j}(\cdot)\right\}$.

$$
R^{y r} \equiv \frac{\left\{(1 / 2) \sum_{\mathbf{s} \in D}\left(Y_{1}^{y r}(\mathbf{s})-Y_{2}^{y r}(\mathbf{s})\right)^{2} / \sum_{\mathbf{s} \in D} 1\right\}^{1 / 2}}{\left|\sum_{\mathbf{s} \in D} Y^{y r}(\mathbf{s}) / \sum_{\mathbf{s} \in D} 1\right|}
$$


where $Y_{i}^{y r}(\cdot) \equiv(1 / 4) \sum_{j=1}^{4} Y_{i j}(\cdot)$ for the $i$-th RCM $(i=1,2)$. The expression (20) is a nonlinear function of the hidden process and expresses the between-model variability as a fraction of the average temperature change over the whole spatial domain.

Since $R^{y r}$ is a function of $\left\{Y_{i j}(\cdot)\right\}$, one can obtain the predictive distribution of $R^{y r}$, given the data, by MCMC (Sect. 4). In this case, the predictive mean of $R^{y r}$ is $4.55 \%$ with a two-sided $95 \%$ prediction interval of $(4.43 \%, 4.67 \%)$. Analogous to $(20), R^{w i}$ is a measure of the between-model variability for winter, and a predictive analysis shows that it is likewise small.

Climate changes produced by RCMs could be used in agriculture to forecast cropvariety yields, in ecology to forecast bird-migration patterns, and in emergency services to forecast bushfire danger. A sobering caveat to these statistical analyses is that there may be common errors made across all RCMs but these errors are unknown. The result would be outputs, $\left\{Z_{i j}^{\text {future }}(\cdot)\right\}$, that give a biased view of what future climate will be like, however current analyses, statistical and otherwise, are unable to account for this.

\section{Acknowledgements}

This research was partially supported by the NASA's Earth Science Technology Office through its Advanced Information Systems Technology Program. We wish to thank the North American Regional Climate Change Assessment Program (NARCCAP) for providing the data used in this article. NARCCAP is funded by NSF, DoE, NOAA, and EPA's Office of Research and Development. Many thanks go to Andrew Holder for his assistance in preparation of this article.

\section{References}

Berliner, L. M. (1996). Hierarchical Bayesian time series models. In Hanson, K. and Silver, R., editors, Maximum entropy and Bayesian methods, pages 15-22. Kluwer Academic Publishers, Dordrecht, NL.

Cressie, N. and Johannesson, G. (2008). Fixed rank kriging for very large spatial data sets. Journal of the Royal Statistical Society, Series B, 70:209-226.

Cressie, N. and Wikle, C. K. (2011). Statistics for Spatio-Temporal Data. John Wiley and Sons, Hoboken, NJ.

Fennessy, M. J. and Shukla, J. (2000). Seasonal prediction over North America with a regional model nested in a global model. Journal of Climate, 13:2605-2627.

Kanamitsu, M., Ebisuzaki, W., Woollen, J., Yang, S.-K., Hnilo, J. J., Fiorino, M., and Potter, G. L. (2002). NCEP-DOE AMIP-II Reanalysis (R-2). Bulletin of the American Meteorological Society, 83:1631-1644.

Kang, E. L. and Cressie, N. (2013). Bayesian hierarchical ANOVA of regional climatechange projections from NARCCAP Phase II. International Journal of Applied Earth Observation and Geoinformation, 22:3-15.

Kang, E. L., Cressie, N., and Sain, S. R. (2012). Combining outputs from the NARCCAP regional climate models using a Bayesian hierarchical model. Journal of the Royal Statistical Society, Series C (Applied Statistics), 61:291-313. 
Kaufman, C. G. and Sain, S. R. (2010). Bayesian ANOVA modeling using Gaussian process prior distributions. Bayesian Analysis, 5:123-150.

Mearns, L. O., Gutowski, W., Jones, R., Leung, R., McGinnis, S., Nunes, A., and Qian, Y. (2009). A regional climate change assessment program for North America. Eos, Transactions American Geophysical Union, 90(36):311.

Nakicenovic, N., Alcamo, J., Davis, G., de Vries, B., Fenhann, J., Gaffin, S., Gregory, K., Grubler, A., Jung, T. Y., Kram, T., La Rovere, E. L., Michaelis, L., Mori, S., Morita, T., Pepper, W., Pitcher, H. M., Price, L., Riahi, K., Roehrl, A., Rogner, H. H., Sankovski, A., Schlesinger, M., Shukla, P., Smith, S. J., Swart, R., van Rooijen, S., Victor, N., and Dadi, Z. (2000). Special Report on Emissions Scenarios: a special report of Working Group III of the Intergovernmental Panel on Climate Change. Technical report, Environmental Molecular Sciences Laboratory, Pacific Northwest National Laboratory, Richland, WA, USA.

Sain, S. R., Furrer, R., and Cressie, N. (2011). A spatial analysis of multivariate output from regional climate models. Annals of Applied Statistics, 5:150-175.

Salazar, E. S., Finley, A., Hammerling, D., Steinsland, I., Wang, X., and Delamater, P. (2011). Comparing and blending regional climate model predictions for the American southwest. Journal of Agricultural, Biological, and Environmental Statistics, 16:586605.

Xue, Y. K., Vasic, R., Janjic, Z., Mesinger, F., and Mitchell, K. E. (2007). Assessment of dynamic downscaling of the continental US regional climate using the Eta/SSiB regional climate model. Journal of Climate, 20:4172-4193. 


\section{Figure Captions}

Figure 1: ESDA of temperature change in North America. (a) Map of $\left\{D^{w i}\left(\mathbf{s}_{l}\right): l=\right.$ $1, \ldots, 11760\}$. (b) Map of $\left\{D^{y r}\left(\mathbf{s}_{l}\right): l=1, \ldots, 11760\right\}$. (c) Plot of $\left\{\left(D^{y r}\left(\mathbf{s}_{l}\right), D^{w i}\left(\mathbf{s}_{l}\right)\right)\right.$ : $l=1, \ldots, 11760\}$.

Figure 2: Video showing linked views of temperature change in North America. (a) SPOT function $T^{w i}(k)$ linked to the map of $\left\{I\left(D^{w i}\left(\mathbf{s}_{l}\right)>k\right): l=1, \ldots, 11760\right\}$. (b) SPOT function $T^{y r}(k)$ linked to the map of $\left\{I\left(D^{y r}\left(\mathbf{s}_{l}\right)>k\right): l=1, \ldots, 11760\right\}$. The video is created by varying $k$ from $1.0^{\circ} \mathrm{C}$ to $7.6^{\circ} \mathrm{C}$ in steps of $0.2^{\circ} \mathrm{C}$.

Figure 3: The NARCCAP pixel $\mathbf{s}_{0}$ that contains NCAR's Mesa Lab is featured. (a) Predictive distribution of $Y^{w i}\left(\mathbf{s}_{0}\right)$ given the data $\left\{D_{i j}(\cdot)\right\}$. (b) Predictive distribution of $Y^{y r}\left(\mathbf{s}_{0}\right)$ given the data $\left\{D_{i j}(\cdot)\right\}$.

Figure 4: Video showing inferential analysis of temperature change in North America. (a) Map of PROT function $\left\{P^{w i}\left(k ; \mathbf{s}_{l}\right): l=1, \ldots, 11760\right\}$. (b) Map of PROT function $\left\{P^{y r}\left(k ; \mathbf{s}_{l}\right): l=1, \ldots, 11760\right\}$. The video is created by varying $k$ from $1.0^{\circ} \mathrm{C}$ to $6.8^{\circ} \mathrm{C}$ in steps of $0.2^{\circ} \mathrm{C}$.

Figure 5: Between-model variability of temperature change in North America. (a) Map of $\left\{D_{14}\left(\mathbf{s}_{l}\right)-D_{24}\left(\mathbf{s}_{l}\right): l=1, \ldots, 11760\right\}$. (b) Map of $\left\{(1 / 4) \sum_{j=1}^{4} D_{1 j}\left(\mathbf{s}_{l}\right)-(1 / 4) \sum_{j=1}^{4} D_{2 j}\left(\mathbf{s}_{l}\right)\right.$ : $l=1, \ldots, 11760\}$. 

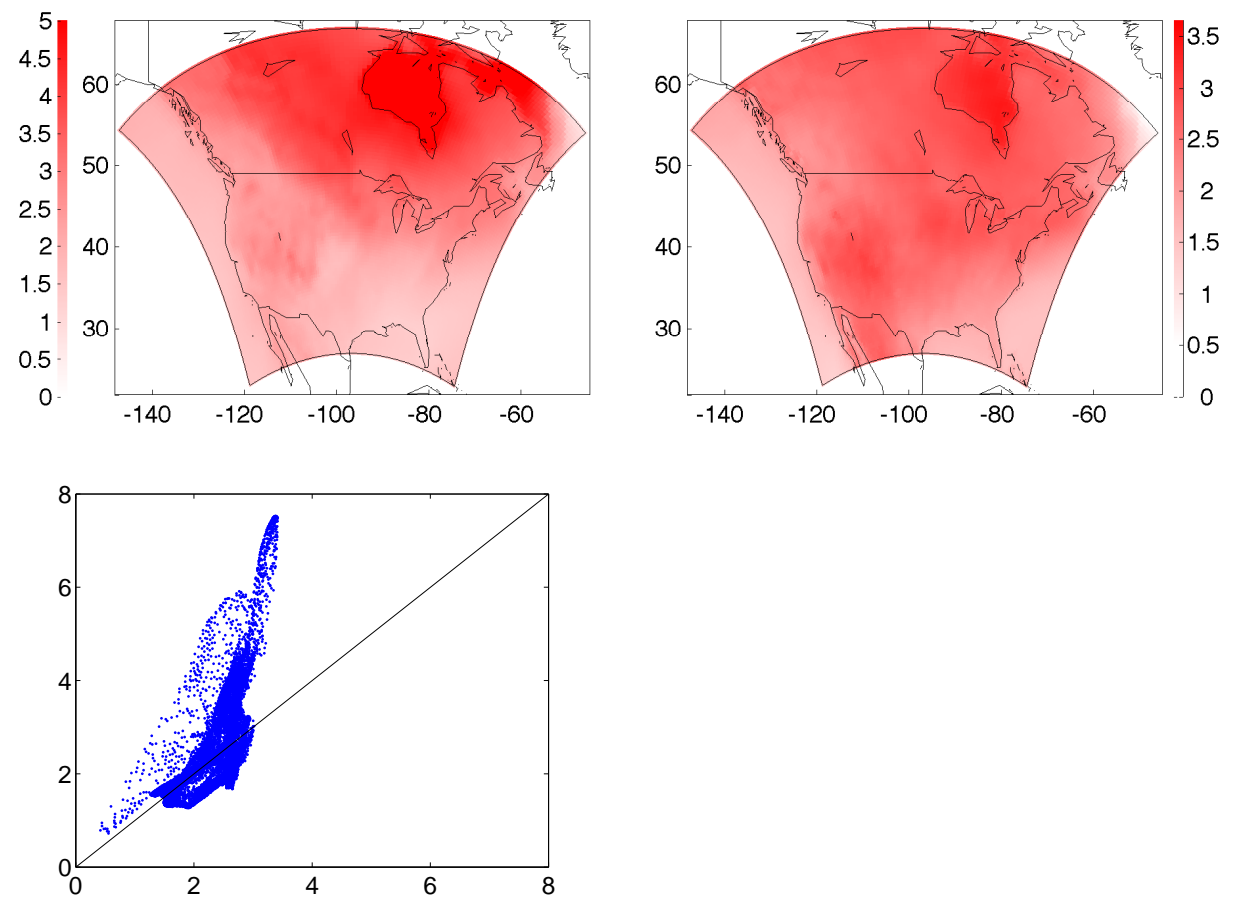

Figure 1: ESDA of temperature change in North America. (a) Map of $\left\{D^{w i}\left(\mathbf{s}_{l}\right): l=\right.$ $1, \ldots, 11760\}$. (b) Map of $\left\{D^{y r}\left(\mathbf{s}_{l}\right): l=1, \ldots, 11760\right\}$. (c) Plot of $\left\{\left(D^{y r}\left(\mathbf{s}_{l}\right), D^{w i}\left(\mathbf{s}_{l}\right)\right)\right.$ : $l=1, \ldots, 11760\}$. 

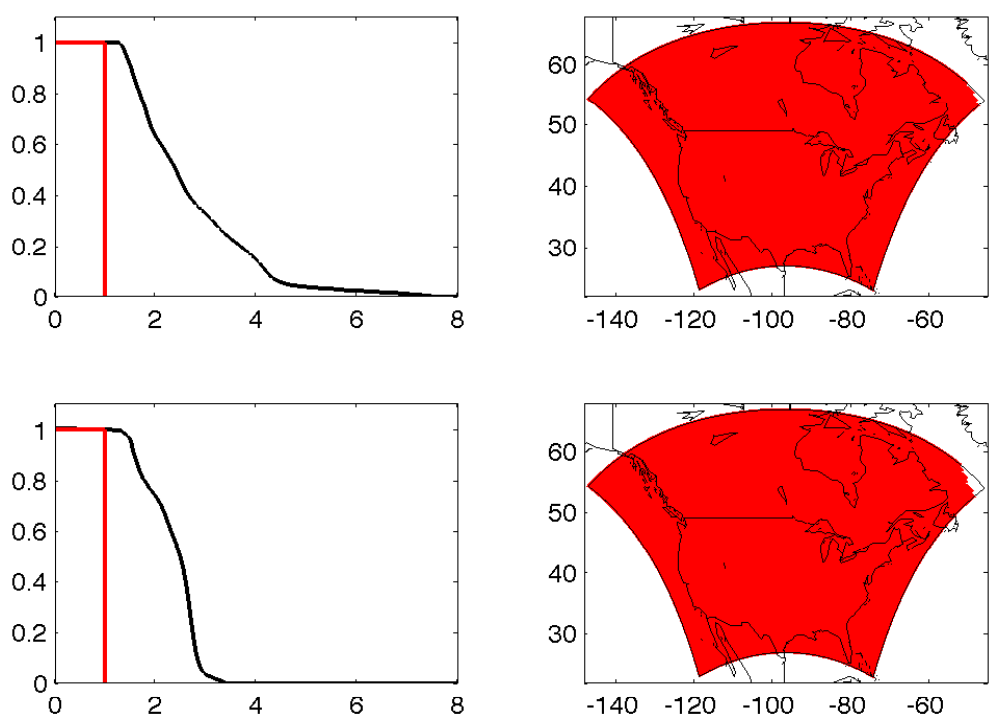

Figure 2: Video showing linked views of temperature change in North America. (a) SPOT function $T^{w i}(k)$ linked to the map of $\left\{I\left(D^{w i}\left(\mathbf{s}_{l}\right)>k\right): l=1, \ldots, 11760\right\}$. (b) SPOT function $T^{y r}(k)$ linked to the map of $\left\{I\left(D^{y r}\left(\mathbf{s}_{l}\right)>k\right): l=1, \ldots, 11760\right\}$. The video is created by varying $k$ from $1.0^{\circ} \mathrm{C}$ to $7.6^{\circ} \mathrm{C}$ in steps of $0.2^{\circ} \mathrm{C}$. 

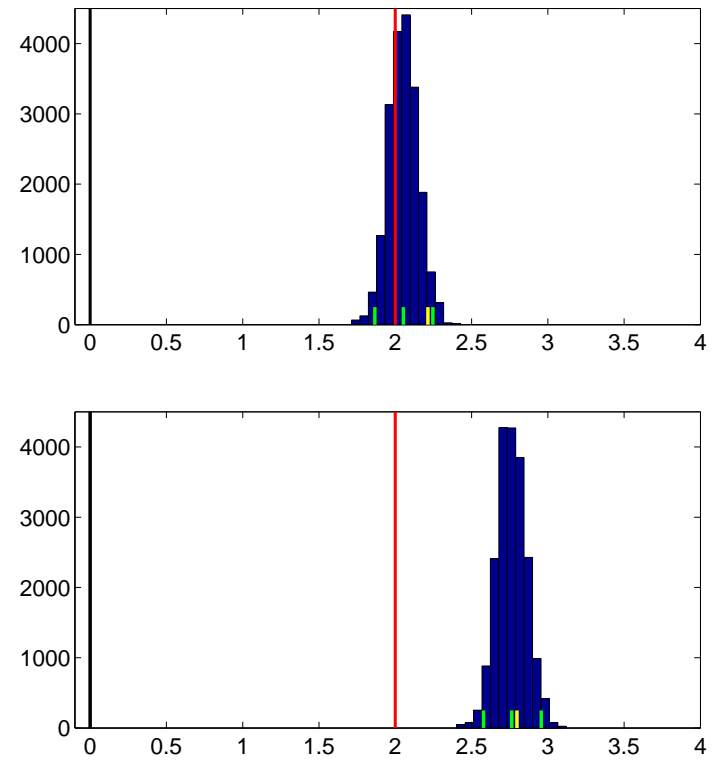

Figure 3: The NARCCAP pixel $\mathbf{s}_{0}$ that contains NCAR's Mesa Lab is featured. (a) Predictive distribution of $Y^{w i}\left(\mathbf{s}_{0}\right)$ given the data $\left\{D_{i j}(\cdot)\right\}$. (b) Predictive distribution of $Y^{y r}\left(\mathbf{s}_{0}\right)$ given the data $\left\{D_{i j}(\cdot)\right\}$. 


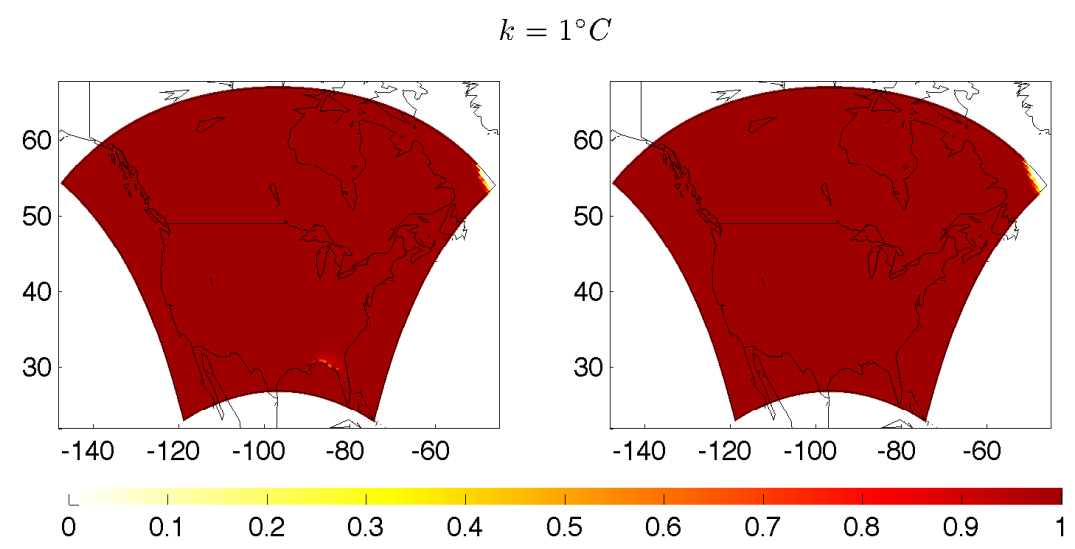

Figure 4: Video showing inferential analysis of temperature change in North America. (a) Map of PROT function $\left\{P^{w i}\left(k ; \mathbf{s}_{l}\right): l=1, \ldots, 11760\right\}$. (b) Map of PROT function $\left\{P^{y r}\left(k ; \mathbf{s}_{l}\right): l=1, \ldots, 11760\right\}$. The video is created by varying $k$ from $1.0^{\circ} \mathrm{C}$ to $6.8^{\circ} \mathrm{C}$ in steps of $0.2^{\circ} \mathrm{C}$. 

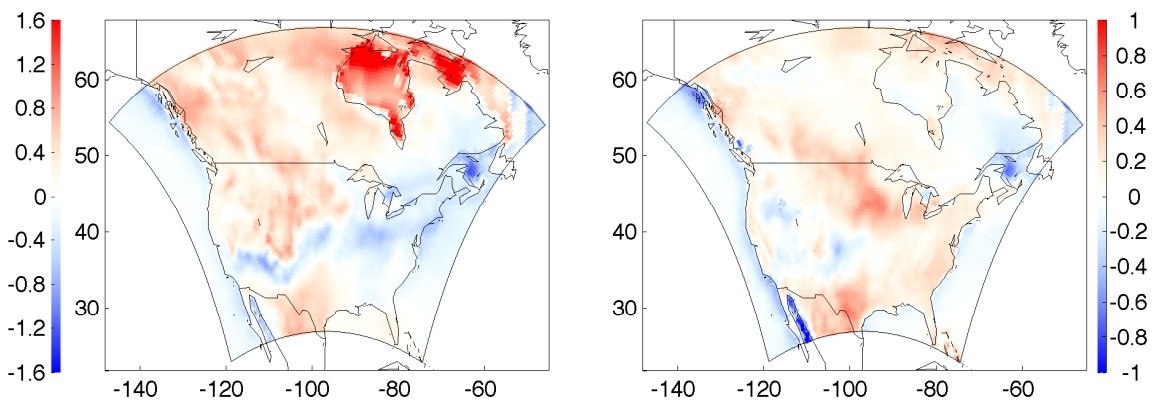

Figure 5: Between-model variability of temperature change in North America. (a) Map of $\left\{D_{14}\left(\mathbf{s}_{l}\right)-D_{24}\left(\mathbf{s}_{l}\right): l=1, \ldots, 11760\right\}$. (b) Map of $\left\{(1 / 4) \sum_{j=1}^{4} D_{1 j}\left(\mathbf{s}_{l}\right)-\right.$ $\left.(1 / 4) \sum_{j=1}^{4} D_{2 j}\left(\mathbf{s}_{l}\right): l=1, \ldots, 11760\right\}$. 